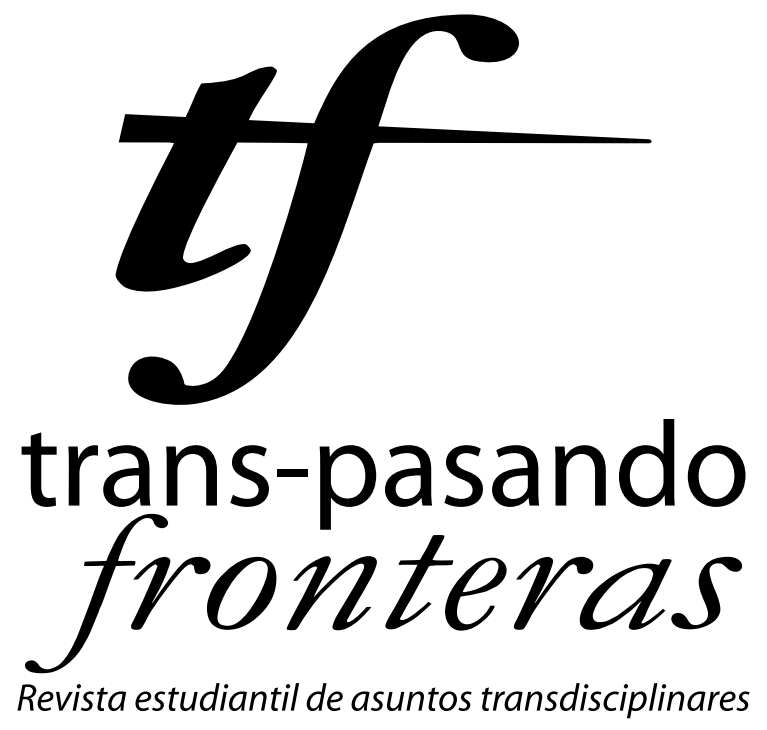

Una publicación de

Estudios

Interdisciplinarios

Jurídicos, Sociales

000 y Humanistas

ICESS

FACULTAD DE

DERECHO Y CIENCIAS

SOCIALES

然 UNIVERSIDAD 


\title{
La deshistorización en el ejercicio del poder capitalista y la dominación comunicativa para la asimetría cultural ${ }^{*}$
}

\author{
Yelina Gómez Martínez ${ }^{* *}$ \\ (yelina.gomez@etecsa.cu)
}

Artículo de investigación científica y tecnológica recibido el 29/07/2012 y aprobado el 18/11/2013.

\begin{abstract}
Como citar este artículo:
GÓMEZ MARTÍNEZ, Yelina (2013). "La deshistorización en el ejercicio del poder capitalista y la dominación comunicativa para la asimetría cultural”. En: Trans-pasando Fronteras, Núm. 4, pp. 117-143. Cali, Colombia: Centro de Estudios Interdisciplinarios, Jurídicos, Sociales y Humanistas (CIES), Facultad de Derecho y Ciencias Sociales, Universidad Icesi.
\end{abstract}

\begin{abstract}
Resumen
El presente trabajo resume la propuesta teórico-metodológica que tiene como estudio a la articulación entre la deshistorización en el ejercicio del poder capitalista y la dominación comunicativa, trastorno

* $\quad$ Este es un artículo que es el resultado del proceso investigativo para optar por el grado de Doctor en Ciencias de la Comunicación Social en la Facultad de Comunicación de la Universidad de La Habana. El proyecto original de investigación se denomina: "La articulación en el ejercicio del poder capitalista y la dominación comunicativa en los intercambios entre modelos de cultura o sociedad".

** Ingeniera en Máquinas Computadoras, graduada del Instituto Superior Politécnico “José Antonio Echeverría” de La Habana. Se desempeña como Especialista Principal del Departamento de Supervisión y Control de la Empresa de Telecomunicaciones de Cuba. Tiene Máster en Marketing y Comunicación por la Facultad de Comunicación de la Universidad de La Habana. Actualmente está en fase de defensa de su tesis doctoral en Ciencias de la Comunicación Social. Desde hace diez años su investigación está encaminada a profundizar en el estudio de la comunicación -desde la semiótica de la cultura- en su articulación inmanente con la cultura, en tanto, modelo de sociedad, lesionada por los efectos de la hegemonía de turno dentro de la mundializada continuidad sistémica del capitalismo.
\end{abstract}


localizable en los flujos comunicativos entre modelos de cultura o sociedad, durante la intersubjetivación entre los individuos bajo la actual hegemonía capitalista. Persigue incluirse en los estudios sobre la comunicación, asumiendo la relación entre el poder y los modos de dominación, utilizando a la semiótica de la cultura como herramienta para estudiar a la comunicación. Integrándonos así, a una tendencia a nivel internacional, que asume la certeza de la interacción entre el poder imperialista y la comunicación.

\section{Palabras claves:}

Sistemas semióticos, supremacía angloestadounidense, asimetría cultura, dominación comunicativa, hegemonía

\section{Introducción}

Durante la expansión histórica de la cultura capitalista, los flujos entre sistemas semióticos se subordinan a la autoproducción burguesa. Lo que se produce, se intercambia, se semantiza y es contenido de la semiosis globalizada, se gesta desde esa autoproducción.

Con respecto al concepto de autoproducción, Acanda (1998) recuerda que el hombre se apropia de la realidad porque la produce. La hace suya al crearla mediante su actividad práctica. Pero el modo en que la hace suya, se apropia de ella, la interioriza y la traduce en elementos de su subjetividad (sus capacidades, potencialidades, ideas, aspiraciones, valores, etc.) está condicionado por el modo en que la produce. Producción y apropiación, por lo tanto, forman un todo indivisible. Producción dice del proceso de objetivación del hombre, que crea los objetos de su realidad y en ellos expresa su subjetividad. Apropiación referencia al proceso de producción de la subjetividad humana, a su auto-producción. Su auto-producción como sujeto. "Todo modo social de producción de la realidad lo es, de de apropiación social de esa realidad (y por lo tanto, de auto-producción del hombre)" (Acanda, 1998: 3).

A contracorriente de ese asunto, no se siguen a pie juntilla procesos de investigación teórica de los espacios "metropolitanos" tradicionales. Tratamos de descubrir mecanismos

1 Estudiosos como Nelly Richard, Luis Martín-Cabrera, Mattelard, Bourdieu, Boaventura de Sousa, Gustavo Lins Ribeiro y Fernando Coronil, entre otros, hacen referencia a un centro de autoridad y poder metropolitano, que define lo que está dentro o fuera de la norma académica y cultural capitalista controlada por esa autoridad del centro de dominio burgués. Tal referencia tiene una implicación instrumental para llamar la atención acerca de tal control, desde las instituciones académicas del primer mundo sobre los espacios antes coloniales; y ahora neocolonizados. Las antiguas metrópolis europeas, reajustaron los procesos de dominación haciéndolo descansar en la preeminencia cultural y comunicativa del conocimiento que imponen las instituciones académicas de las antes metrópolis y en la actualidad integrantes del sujeto social (colectivo) capitalista. Nota de la autora (NA). 
y estrategias teóricas que dilucidan al control del poder burgués, deshistorizado en su ejercicio, para la dominación comunicativa a partir de esa autoproducción.

Nos soportamos en la semiótica de la cultura, entendiendo que la producción social de signos, las posibilidades y normas de significación y semantización, así como el fundamento cultural del orden político de una sociedad dada, están dilucidados por un sistema modelizante de primer nivel o lengua. La lengua anglófona, desde su variante estadounidense ${ }^{2}$, es dominante desde el punto de vista comunicativo.

La noción del sentido impuesta por la globalización cultural angloestadounidense -de su sistema modelante de primer nivel en hegemonía-, interviene en los modos comunicativos entre los individuos y entre culturas. La comunicación estudiada desde dimensiones de las academias metropolitanas, o fuera de ellas, pero siempre subordinada a sus normas no ha hecho más que intentar una y otra vez metodologizar -buscando legitimación científica- a la dominación comunicativa. En este proceso tiene un rol esencial la deshistorización en el ejercicio del poder capitalista vigente, invisibilizado a través de mucha de esa teoría sobre la comunicación.

El sujeto histórico, a través de la comunicación, no puede pasar solo como un "artificio" perceptivo. La evidencia está en la sumisión cultural a las relaciones de dominación. Crear redes de sometimiento a un centro (canon cultural) en expansión, ha sido y es aún la finalidad de esa deshistorización burguesa.

Para el análisis de esta articulación entre la deshistorización del ejercicio del poder capitalista y la dominación comunicativa, partimos de los métodos del pensamiento teórico lógico. En tal sentido, la semiótica de la cultura -desde la Escuela de Tartu y sus seguidores que la han ido actualizando con el objetivo de estudiar al poder- utilizada como herramienta metódica para estudiar a la comunicación, aporta resultados efectivos con implicación categorial. La interacción entre saber mutuo y memoria común -dos conceptos provenientes de la semiótica de la cultura-, que se dilucida en la comunicación, permite confirmar que lo que los sujetos intercambian es historia cultural. La filosofía marxista para la praxis es el soporte para contrarrestar a la unipolaridad capitalista en su control sobre los procesos comunicativos.

2 Una variedad o variante de una lengua es una forma que difiere sistemática y coherentemente de otras formas de la misma lengua. (NA). 
Al investigar a la articulación entre la deshistorización en el ejercicio del poder capitalista y la dominación comunicativa mediante un análisis crítico sobre la comunicación desde la semiótica de la cultura, se confirma la hipótesis de que la deshistorización en el ejercicio del poder capitalista como proceso y la dominación comunicativa como su controlador -o driver-ineludible, se sirven de la supremacía codicial angloestadounidense ${ }^{3}$ que deforma los "textos" y con ello la comunicabilidad entre los modelos de cultura o sociedad, provocando trastornos convenientes que benefician a la autoproducción burguesa, durante su hegemonía.

De ahí que el estudio se concentre también en una reflexión crítica sobre la interacción entre modelo de cultura o sociedad y acumulación histórica o totalidad cultural. Esa acumulación provee al modelo de sociedad de los contenidos (selectivos) necesarios, que se intercambian y actualizan mediante sistemas semióticos o comunicativos durante la intersubjetivación entre los individuos, esencial a la autoproducción.

La supremacía codicial angloestadounidense se convierte en fiscalizadora intrusiva de los contenidos comunicativos a nivel global, mediante su sistema modelante de primer nivel: la lengua inglesa en su modelización angloestadounidense. ${ }^{4}$

Según Lotman (2003), un texto es para la Escuela de Tartu un signo con más de un significado por su importancia dentro de una cultura, y para ello debe estar codificado, como mínimo, dos veces. Lo que esa supremacía codicial deforma "textualmente” está, de una manera u otra, contenido dentro de la deshistorización que perturba a la comunicación.

El proceso de la deshistorización en el ejercicio del poder capitalista -que se articula con la dominación comunicativa- lo asumimos desde los resultados de investigación de Néstor Kohan, básicamente, durante su estudio enjundioso del pensamiento y la obra de Carlos Marx. Dicha articulación posee implicación metodológica, en tanto este proceso es improbable sin un modo de dominación que le abra espacio en la reproducción (social).

3 La lengua o sistema modelizante de primer nivel contiene al código de una cultura mediante sistemas semióticos al uso. El código inglés se prorroga hegemónico en la variante angloestadounidense a partir de 1944, y domina por supremacía codicial a la interacción entre culturas. (NA).

4 Reconociendo al sistema de la cultura como resultado del sistema de modelización primario y, a su vez, como conjunto de sistemas culturales o conjunto de sistemas de modelización secundarios, que en interacción con la actual supremacía codicial, dilucida o intenta dilucidar a los procesos de intersubjetivación, perturbando el contenido de los sistemas semióticos o comunicativos. (NA). 


\section{Los flujos comunicativos entre modelos de cultura o sociedad para definir a la dominación comunicativa}

La necesidad de reducir la indeterminación del mundo humano lleva a los sujetos a diseñar, representar y compartir - a posteriori- una "copia simplificada, traducción abreviada" (Navarro, 2007: 70) que al intercambiar saber mutuo y memoria común, constituya, describa y actualice a un determinado modelo del mundo para la implementación sistémica de programas para el individuo y la colectividad; y establecer la elección de los actos para influir sobre el mundo, así como las reglas de estos actos y sus motivaciones, en tanto técnicas sociales de reacción significante a una situación histórica.

A través de la semiosis o producción social de signos, se diseñan y actualizan sistemas semióticos para que los individuos se subjetiven dentro de un modelo de cultura o sociedad; y a posteriori, compartan -comuniquen- y actualicen esa modelización de su sociedad -su cultura- e intelijan a otros modelos (Gómez, 2011b).

¿Qué relación tiene la cadena anterior con la postura teórica que asumimos? Todo conjunto de sistemas semióticos, comunicativamente en uso, cumple dos funciones, bien definidas dentro de una colectividad dada en un contexto histórico específico, "no es sólo un producto social, sino también una práctica social" (Navarro, 2007: 71). Lo que se intercambia son signos (de productos y prácticas sociales), insertos en sistemas semióticos para darles sentido y compartirlos mediante la "bisagra” entre la acumulación cultural y el modelo de cultura o sociedad en los intercambios entre sistemas semióticos o comunicativos.

La supremacía codicial angloestadounidense y su dominación sobre mecanismos de regulación comunicativa

La lengua es la convención social que se adopta para ejercitar la facultad del lenguaje y constituye, ante todo, un código de carácter social, es decir que el lenguaje "permite la comunicación social y, con ella, la reproducción de las relaciones sociales” (Romano, 2005: 4). Desde la semiótica de la cultura podemos definir que la lengua (idioma), en tanto sistema modelizante o modelante de primer nivel y depositaria gramatical del código, determina la sintaxis y la semantización en una cultura a través del conjunto de sistemas semióticos que la soportan. Por la importancia de los sistemas modelantes de primer nivel, precisamos que un sujeto cultural no aprende simplemente de un lenguaje 
cierta colección de fórmulas y recursos comunicativos y expresivos sino que aprende a actuar y a interactuar en él, ya que "el sujeto se define a través de su operar (y aprender a operar) en los dominios lingüisticos en que participa" (Lampis, 2008: 59-60).

Lo anterior es válido porque, si tenemos en cuenta que "ni siquiera un "lenguaje" gestual, puede significar, indicar o expresar sin la mediación de un código pre-existente” (Spivak, 1996: 20), comprenderemos que la supremacía codicial referida perturba a los flujos entre culturas para inducirles entropía "incoercible" fuera de fundamento burgués, versus relación simétrica inter/códigos. El cambio es siempre hacia y desde el código hegemónico que deviene en el "traductor" durante los intercambios entre las culturas.

A contrapelo del enredo epistémico que impone la supremacía codicial de la actual hegemonía capitalista, los investigadores González y González -desde una perspectiva histórica- lo califican de "imperialismo lingüístico":

presente [...] a través de la forma, como a través del contenido. La lengua dominante da lugar a una forma oculta pero eficaz de discriminación, establece una división social entre individuos y grupos bajo el criterio del lenguaje, distinguiendo entre quienes tienen acceso en grados diversos al lenguaje dominante y quienes lo ignoran (González y González, 2005: 1).

Esto es válido para todas las lenguas dominantes en cada época de la historia del sistema capitalista y de la expansión de su cultura. Un análisis crítico de la dominación comunicativa discierne a la guerra cultural, como lógica imperialista, en toda la línea, que moviliza formidables instrumentos y recursos, y ejerce controles totalitarios sobre la información, la formación de opinión pública, los gustos y los deseos (Martínez Heredia, 2003). Y se dirige a impedir la producción de voluntades, identidades y pensamientos opuestos a la dominación, buscando la deformación, y posterior extinción codicial de sistemas comunicativos y culturas que éstos soportan, e incluye al dominio de una lengua sobre las otras en pos de un genocidio lingüístico, la desaparición progresiva de cientos de lenguas, con toda la riqueza cultural que contienen, y que no la causa solamente el inglés, sino también otras lenguas "mayoritarias", normalmente consideradas lenguas "nacionales", impuestas mediante formas más o menos sutiles por diversos Estados metropolitanos y favorece obviamente a las élites, que no solo 
suelen ser las que usan las lenguas "nacionales", sino también las que pueden acceder al aprendizaje del inglés (González y González, 2005).

Las mayorías, en asimetría comunicativa, cuando logran mantener su lengua, se ven privadas de su identificación plena con la misma, lo que conlleva a un empobrecimiento cultural: por una parte, a la falta de dominio de la lengua propia, y por la otra, a un incesante préstamo de términos foráneos que en muchas ocasiones son innecesarios. Esto se evidencia en los flujos entre modelos de cultura e individuos subjetivados que los representan, y entre modelos y subjetividades diferentes.

Los trastornos que provoca el código angloestadounidense, durante la perturbación de "textos" mantienen operativa a la dominación para una "neutralidad", que invisibilice a su supremacía codicial sobre los mecanismos de regulación comunicativa indispensables. La competencia sígnica, (nivel sintáctico) que permite la decodificación, se explica a partir del reconocimiento de las estructuras que norman y jerarquizan los elementos, capaces de construir el sentido y que permiten acceder al significado depositado en toda producción textual. La competencia cultural (nivel semántico), que supone la activación de las selecciones contextuales y circunstanciales dotan de significado a la estructura sintáctica. Ambas competencias, según Tenoch Cid (2006), actúan como mecanismos reguladores que permiten entender a todo texto como una serie de inferencias para recuperar la información depositada en su interior. La combinación necesaria de ambas competencias debe ser capaz de generar esquemas cognitivos que, a su vez, extenderán su eficacia ante los cambios que la comunicación globalizada exige.

La deformación de "textos" por parte del código hegemónico angloestadounidense implica la imposición de su gramaticalidad desde su supremacía codicial, obviando cualquier consenso coherente en los procesos inter-códigos.

La cultura del capitalismo en su expansión histórica no se relaciona con una Cultura -civilización-o acumulación cultural en específico. De ahí que para dominar en supremacía codicial a modelos de cultura o sociedad, interactúe con la cotidianidad de éstos mediante el trastorno de los "textos" en los flujos comunicativos durante la reproducción de sus relaciones de producción.

A partir de lo anterior podríamos inferir que Colón y Cortés fueron pioneros 
comunicativos de la modernidad occidental. La deformación de "textos" que contienen sistemas semióticos o comunicativos, esencialmente originarios de culturas no euroccidentales, podría tener su génesis temprana en el control de espacios signo/ semánticos durante las etapas de conquista previa; y no sólo en la continuidad de la supremacía codicial anglo. Gracias a esa expansión histórica de la cultura del capitalismo a lomos de la modernidad europea (Amin, 2010).

La supremacía codicial de una hegemonía históricamente contextualizada está, aún, en función de la expansión histórica de la cultura del capitalismo hacia la sistémica política de un orden dominante en la desigualdad, a través de la hegemonía capitalista del modelo de cultura o sociedad de turno; pero sin vínculo con una acumulación o Cultura (civilización) determinada.

Según Gómez (2011a), una acumulación cultural interactúa con un modelo de cultura o sociedad. El modelo consensua intersubjetivamente con dicha acumulación su equilibrio e interacción en su interior, y con otros modelos, mediante sistemas semióticos en un contexto histórico específico. Martínez Heredia define al sentido con que podemos entender la acumulación cultural cuando expresa que:

\begin{abstract}
La cultura nacional alberga y expresa una riqueza de rasgos y elaboraciones propias, hechas con los más disimiles materiales y modos, por los más diversos grupos sociales, en depósitos sucesivos y simultáneos. Esa acumulación cultural es la que opera en cada época y en cada coyuntura; en ella se inscriben todos los aspectos y casos particulares, con sus complejos de relaciones e interacciones (Martínez Heredia, 2001: 68).
\end{abstract}

A través de los sistemas semióticos -en tanto comunicativos- que soportan a un modelo de cultura, los individuos subjetivados recurren a dicha acumulación para seleccionar elementos que diseñan y actualizan a ese modelo de cultura o sociedad, que además describen, representan y comparten dentro de su sociedad y en interacción inmanente con otras, utilizando a esos sistemas semióticos.

Con respecto a la categoría modelo, Sonesson (2002) en su trabajo sobre semiótica de la cultura ha precisado dos cosas que al parecer han fueron olvidadas por la misma escuela de Tartu y sus sucesores: que no se trata de la Cultura por sí misma -acumulación histórica-, 
sino del modelo (cotidianidad o cultura) que los sujetos hacen de su Cultura; y este modelo mismo tiene más que ver con las relaciones entre culturas (cotidianidades) que con las Culturas (acumulaciones históricas). En la semiótica de la cultura, una cultura es entendida como un mecanismo para producir "textos" y también para excluir o deformar" "textos" de otras culturas; y comprender que "lo que los norteamericanos divulgan son "textos" sacados de otras culturas y deformados" (Sonesson, 2002: 117).

Podemos resumir que el estudio de los modelos de cultura, mediante el diseño a posteriori, actualización y flujos entre sistemas semióticos que soportan a los modelos en un contexto histórico determinado, tienen que ver más con el intercambio entre modelos que con la génesis de una Cultura -acumulación histórica, o totalidad- con la que interactúa un modelo, con los procesos de intersubjetivación entre los individuos que los representan, y sobre todo con la deformación de "textos" de otros modelos y del suyo propio, por parte del código angloestadounidense, antes de hacerlos circular.

\section{Saber mutuo y memoria común.}

A los individuos subjetivados en distintos modelos de cultura -y a los mismos modelospara comunicarse no les es suficiente "saber" cada uno por su cuenta, sino que les resulta imprescindible compartir un saber mutuo hacia una memoria común, que constituye, organiza, sistematiza, a un saber mutuo garante de posibilidad comunicativa.

La comunicación no se agota en el proceso de transmisión de información, sino que es un primer paso, hasta ahí inacabado, porque se extiende para su materialización a ubicar una información en un contexto histórico cultural determinado, haciendo operativo a ese saber mutuo que implica a una memoria común. Es decir, la "competencia comunicativa e interaccional" es consecuencia de ese saber mutuo, y cualidad subjetivo-cultural históricamente contextualizada, en pos de confirmar que se comparte de forma comunicativa -que es la única posible en tal proceso- una memoria común.

¿Cuál es la importancia de un saber mutuo y una memoria común (su manipulación) para los objetivos de la dominación comunicativa bajo la hegemonía angloestadounidense? El control de las diferencias, en desigualdad, para la subordinación comunicati-

5 Este es el tipo de deformación al que nos referimos desde la perspectiva semiótica. 
va, cultural, que se dilucide en el trastorno de esa memoria común y saber mutuo, compartidos mediante los flujos entre sistemas semióticos, porque la comunicación entre los diversos sin saber mutuo ni memoria común traería, devolvería, conllevaría a "lo mismo" una y otra vez por ausencia de dinámica cultural impredecible. Da y devuelve la misma cosa, a partir de que "si emisor y destinatario ${ }^{6}$ estuvieran dotados de códigos iguales ${ }^{7}$, la comprensión entre ellos sería perfecta, pero el valor de la información transmitida sería mínimo y la misma información rigurosamente limitada. La comunicación normal lleva implícita (como sostiene Lotman) el supuesto de una no identidad de partida entre el hablante y el oyente" (Lozano, 1995: 3).

La memoria común se comparte porque es sistémica. El saber mutuo resulta de la interacción entre los diversos desde esa memoria común, hoy global en reciprocidad - o nocomunicativa. Se comunica lo que no se conoce, para conocerlo y aprenderlo. Lo que se conoce, se intercambia para comunicarlo como saber común.

La transmisión de información, reiteramos, no es más que un primer paso, atraviesa al conocimiento, históricamente contextualizado, pasando por el logro de compartir, comunicarse un saber mutuo hacia una memoria común, que es histórico/cultural, acumulativa, sistémica. Lo que equivale a la capacidad comunicativa de actualizar y compartir a una memoria común mediante saber mutuo. El control de tales procesos en los flujos entre sistemas semióticos es garantía para la fiscalización de sus contenidos. Es decir, hegemonía comunicativa, por cultural, desde el poder.

La memoria común actualizada y compartida comunicativamente mediante saber mutuo es lo que propicia una subjetividad dueña de "competencia interaccional" para re-construir la información transmitida, procesarla hacia el conocimiento. No existe transmisión pasiva (abstracta) de información. Codificación, decodificación y semantización son inmanentes.

Lo que modelos e individuos subjetivados comparten, se comunican mutuamente, es historia cultural -saber mutuo dentro del modelo y entre modelos- contextualizada mediante los flujos comunicativos hacia una memoria común, indispensable para facturar cualquier acción comunicativa. Es decir, todo modelo de cultura es una reducción

6 Términos que en la actualidad se complican en su rol, al asumir los flujos entre sistemas comunicativos, por su globalidad, una dimensión circular indetenible. (NA).

7 Por representar al mismo modelo. (NA). 
selectiva que los individuos subjetivados dentro de una cultura hacen de su sociedad y de esa acumulación o totalidad de su historia cultural.

Desde la semiosis, los sistemas semióticos diseñan, describen y hacen circular, en tanto comunicativos, lo que los individuos subjetivados entienden que deben compartir (comunicar), actualizar, representar en tanto esencia que da sentido a la sociedad que comparten en un contexto histórico determinado. No es todo el contenido de la acumulación cultural lo que conforma el modelo, sino un resumen selectivo -históricamente contextualizado- de esa acumulación.

El modelo angloestadounidense (su hegemonía) ha impuesto a través del conjunto de sistemas semióticos que lo soportan una descripción selectiva de "textos" perturbados a tenor de sus intereses de poder, con fundamento cultural burgués, que dilucidan al orden político imperialista como el "vencedor" comunicativo, aún en estatus de expansión global. Desde los sistemas semióticos que soportan a ese modelo, se propaga una perspectiva fetichista de su sociedad en la comunicación de su cultura.

\section{La dominación comunicativa}

Las culturas son signo/simbólicas y sistémicas. Invocando a su modelo, una cultura, mediante supremacía codicial por sobre las otras, puede extender su dominación tanto como lo implique la expansión de su hegemonía; y dicha expansión es, esencialmente, comunicativa. Incluye a la vigencia histórica de su conjunto de sistemas semióticos para la intersubjetivación de los individuos que la representan. También a los procesos de conquista política, control de un saber mutuo y memoria común, para lo que deba inferirse como dentro o fuera de éstos:

\footnotetext{
La adaptación semiótica a las reglas de una civilización externa suele estar ligada a la dicotomía "cultura/ barbarie". Los antiguos griegos llamaban bárbaros a los persas y egipcios, que les superaban por la riqueza de su tradición cultural [...] En fin, cabe aquí recordar el dictum de Montaigne (1,31): "Cada cual llama barbarie a lo que no forma parte de su costumbre" (Lozano, 1998: 4).
}

El control sobre los sistemas semióticos fue pugnado y consensuado, esencialmente, mediante dos maneras: por referencia e inferencia (Sonesson, 2002) desde una posición 
dominante. Fue la inmanencia comunicativa que adquirió la incesante acumulación de capital desde la temprana globalidad capitalista, en los espacios conquistados y colonizados, mediante la diversidad de opciones en la dominación, con mucha recurrencia a la coerción que es también un modo comunicativo, que devino en hegemonía con extensa sucesividad histórica, apelando al control y al trastorno de sistemas comunicativos.

Para el capitalismo moderno, es de gran importancia la dominación psicológica del individuo y la manipulación de su conciencia y de sus necesidades, porque mientras que el mundo interior del individuo cada día es más dominado por la industria de la comunicación masiva, o de la conciencia, de la cultura o del entretenimiento. La comunicación se ha convertido en un sector estratégico en el plano político, cultural y económico (Romano, 2004).

Sánchez (2010), en su análisis, tiene puntos de coincidencia con Romano y, aunque centra su mirada en los mass media, conceptualiza la dominación comunicativa (culturalideológica) del Tercer Mundo como una parte de la dominación económica y además un requisito para la misma, donde las grandes multinacionales de la electrónica y el audiovisual, principalmente norteamericanas, dominan los mercados en América Latina y buena parte de África. Porque "el nuevo colonialismo ya no busca la conquista del territorio, sino de los mercados, de las bolsas, para lo cual necesita dominar el espacio simbólico de los medios de comunicación y de las conciencias" (Sánchez, 2010: 1).

Precisa Gómez (2011b) que la interacción entre sistemas semióticos o comunicativos devino en instrumento para la producción de realidad y autoproducción burguesas durante la expansión histórica de la cultura del capitalismo, que hoy busca re-colonizar al mundo humano reductible ${ }^{8}$. Proceso al que él discierne como: dominación comunicativa; ubicable, hoy, en la guerra cultural.

\section{La deshistorización en el ejercicio del poder capitalista}

En principio, no existe probabilidad efectiva de dominar comunicativamente a los individuos durante el proceso de intersubjetivación caro al de autoproducción, en la emergencia de una sistémica social, sin desactivar o deformar la acumulación histórica con la que debe,

8 La función de la cultura, vista desde una perspectiva semiótica, consistiría en reducir la indeterminación del mundo humano. (NA). 
selectivamente, interactuar un modelo de cultura o sociedad, en pos de su fundamento históricamente contextualizado. El vínculo entre las relaciones sociales de producción y el poder, que constantemente se comunica en su ejercicio con la actividad práctica, cotidiana de los individuos, depende de la dominación de la interacción entre ellos.

Desde la semiótica de la cultura, hemos dilucidado que el sistema modelante de primer nivel, la variante estadounidense del inglés tiene la supremacía codicial vigente a nivel global. Su hegemonía cubre a las relaciones sociales de producción capitalista. Lo que se semantiza, desde una diversidad abrumadora en la producción social de signos, también a nivel global, está dominada por esa supremacía codicial. Manipular a la capacidad de discernimiento crítico posible a comunicar, borrando cualquier rastro de acumulación cultural para sustituirla por un presentismo, históricamente amnésico porque:

\begin{abstract}
deshistorizando el presente, los ideólogos de las clases dominantes nos inducen a las nuevas generaciones a no mirar hacia atrás. Sin historia, detrás de nosotros quedaría el vacio, el desierto helado de la memoria, empezaríamos de cero. Este horror cotidiano se volvería eterno. Enfrentando esa meditada operación ideológica se nos impone recuperar nuestras tradiciones. [...] Durante mucho tiempo el Che Guevara, [...] fue clandestino, subterráneo, innombrable. Luego fue nombrado, por una operación de [deshistorización] [...], hasta el hartazgo. Pero siempre con la misma intención: esconderlo y ocultarlo para que las nuevas generaciones no se apropien de su ejemplo (Kohan, 2011: 274).
\end{abstract}

Al discernir la aplicación práctica del proceso de deshistorización en el ejercicio del poder capitalista en articulación con la dominación comunicativa, confirmamos que, aun cuando ha sido dicho proceso copiado por la variante del socialismo "real" estalinista, es oriundo del pensamiento liberal reformado a partir de 1848, como resultado de la conversión de la clase burguesa, en el poder, en conservadora. El objetivo es la eternización del sistema, por una parte, y la invisibilización de su poder por la otra.

La conservación de la propiedad privada y la misma definición de la categoría ciudadano a partir del individuo propietario, requerirían para su conservación de esa invisibilidad del Estado y el poder como, principalmente, modo de ordenamiento social. Ello nos permite enunciar que para la despersonalización del poder, partiendo del modo liberal de pensar la realidad, para que el poder sea legítimo tiene que pensarse como poder $a b s$ - 
tracto. "Se fijó una visión del Estado y el poder que los representa como desvinculados de todo nexo concreto con intereses o grupos especificos, como máquina cuyo solo propósito es la conservación del orden" (Acanda, 2002: 212).

Ese objetivo hubiese sido improbable sin recurrir a la deshistorización en el ejercicio del poder capitalista, mediante la dominación comunicativa. El problema de la invisibilización del poder capitalista, en su ejercicio, se explica a partir de las deformaciones que alienan al discernimiento crítico de las relaciones sociales; es decir, del propio poder como una relación social "localizable" solo en la semantización fetichista del Estado, que es también un modo de relación social, camino de eternizar a la misma deshistorización, y que devino lamentablemente en discernimiento de un tipo de marxismo. Resulta ya un lugar común, para el marxismo economicista, situar el poder solo en el aparato de Estado, porque allí están las armas, de donde se colige que el poder se ha semantizado habitualmente a partir de las armas. Pero el aparato de Estado, las armas, las Fuerzas Armadas, etc., son la emergencia del poder, e inmediatez de lo que se ve, lo "observable". Como el pensamiento de Marx es una invitación permanente a sospechar de lo inmediato y a aguzar la vista por detrás de lo "observable", urge averiguar qué hay más allá de estas "cosas" e "instituciones" visibles (Kohan, 2007).

La misma dominación comunicativa consolida la omisión de que lo que esas "cosas" ocultan es la existencia de relaciones donde reside el poder, donde situarlo y tratar de asirlo conceptualmente. Ni en la esfera de la relación ser humano-cosa (núcleo del fetichismo y la cosificación), ni en el de la relación ser humano-naturaleza (espacio teórico en el que se mueve el materialismo metafísico de los ilustrados franceses del siglo XVIII o el DIAMAT soviético en el siglo XX) sino en aquella otra anchura constituida por las relaciones de los seres humanos entre sí. Así como Marx descubre en su crítica de la economía política que detrás de "las cosas" económicas están las relaciones que han sido reificadas y cristalizadas en el proceso del fetichismo (Kohan, 2007), igualmente podríamos pensar que detrás de estas “cosas" (por ejemplo, las armas) lo que hay no es más que relaciones de poder entre las personas.

Kohan (2007) expresa que en tanto modo de relación social, el ámbito del poder es aquel donde se producen, se constituyen y se reproducen las relaciones sociales, el ámbito donde se forman y se realizan (a partir de relaciones anteriores) relaciones sociales, luego de lo cual tiene lugar su proceso de reproducción. 
Es necesaria una precisión -en ocasiones olvidada- que ha extendido "lagunas" hacia disciplinas como la comunicación, desde el mismo espacio socialista de los procesos sociales, que deben ser dilucidadas al margen de epistemologías neutrales y continencias reflexivas dentro de la dimensión académica. Porque a diferencia de lo presupuesto por la vulgata economicista del estalinismo, las relaciones sociales se conforman según la teoría marxiana de El Capital, la cual afirma que a partir de confrontaciones y contradicciones entre los sujetos históricos intervinientes, es decir, a partir de relaciones de fuerza entre los polos. En este sentido, deberíamos (re)pensar la categoría que expresa la relación social de capital como una relación de fuerza entre los dos sujetos sociales involucrados: el capitalista (colectivo) comprador de la fuerza de trabajo y el obrero (colectivo) vendedor de esta última. Aquí dentro, al interior de la relación misma, reside la lucha de clases entre un sujeto social dominador y otro sujeto social dominado (que puede rebelarse y trastocar la relación social de capital) (Kohan, 2007).

A tenor de lo anterior se plantea que tangencialmente "el poder constituye el ámbito de las relaciones de fuerzas -que atraviesan tanto las relaciones sociales como las relaciones politicas-donde se producen y reproducen nuevas relaciones a partir de la ruptura de las anteriores" (Kohan, 2007: 19). Para interrogarnos después: ¿en qué consiste esa ruptura?, si no es en el surgimiento de una diferencia cualitativamente sustancial en un ámbito, un corte, una discontinuidad, un trastrocamiento, un salto (Kohan, 2007). Pero de inmediato el filósofo argentino aclara, que en cuanto a la formación de relaciones sociales, ésta implica la construcción de una nueva relación antes inexistente. Si hemos concebido a las relaciones sociales como relaciones de fuerza, con un enfrentamiento inherente que las atraviesa intrínsecamente, deberíamos entonces deducir que "una nueva relación" "es el producto de una confrontación anterior porque las nuevas relaciones sociales que surgen no "flotan" en el presente; son el punto de llegada desde un proceso previo de enfrentamiento, donde uno de los polos ha sido derrotado antes, y llega vencido al proceso de formación de la nueva relación social" (Kohan, 2007: 19).

Lo que podría explicar la subordinación comunicativa, por cultural, que los "vencidos" bajo la supremacía angloestadounidense experimentan, cuando los resultados de la producción social de signos (semiosis), proveniente de los márgenes del centro de la dominación capitalista, al acceder algunos de sus elementos hacia ese centro de dominación, sufren una 
re-semantización deshistorizada, impuesta por el modo de dominación que se articula con el proceso en estudio.

Lenguas, culturas y naciones emergentes tras la expansión histórica de la cultura del capitalismo a lomos de la modernidad europea experimentan ese proceso en diversidad de gradaciones. Porque el vencido (que no es un individuo particular y aislado, sino (un sujeto) clases sociales, fuerzas sociales aplastadas en la lucha de clases) no tiene otra opción que formar parte de esa nueva relación que el vencedor lo obliga a constituir. Y después de mucho tiempo transcurrido, el vencido "olvide" esta confrontación inicial y su derrota previa en el enfrentamiento, entonces piense que ha formado parte de esa relación en forma "libre" y "voluntaria", por una decisión racional, autónoma, soberana y autoconsciente. Esa es "la actitud del sujeto moderno contractualista presupuesto por la economía politica neoclásica y su racionalidad calculadora e instrumental”, (Kohan, 2007: 19).

La dominación comunicativa activa al proceso de deshistorización del poder capitalista, en la "eternización” del sistema burgués cuando "la 'realización' de ésta constituye una producción de relaciones como resultado de la victoria en la confrontación. Un punto de alcance de una nueva etapa en el proceso posterior al triunfo, o sea: la construcción de un dominio estable. En otras palabras: la paz, momento estratégico de la confrontación y al mismo tiempo resultado de la victoria previa en el enfrentamiento” (Kohan, 2007). Si la derrota es tal que no se visualiza en el campo de los "observables" ninguna posibilidad de revertirla, los sujetos sociales dominados y vencidos empiezan a otorgar consenso al vencedor y a "olvidar" el turbio origen de la paz, autorrepresentándose imaginariamente la situación posvictoria como una relación eterna, sin origen y sin futuro. "Deshistorizar el ejercicio del poder, he ahi la clave para su reproducción [capitalista]" (Kohan, 2007:20).

Para finalizar con la definición de la deshistorización del poder es indispensable no olvidar que cuando se alude a las relaciones sociales capitalistas como algo eterno, ahistórico, o como consecuencia de la voluntad mutua de los capitalistas y de los trabajadores, o también como producto exclusivo de relaciones económicas, estamos en el plano de la apariencia, de lo que se ve, de lo observable, de lo accesible a los sentidos y a la conciencia inmediata y precrítica al sentido común previamente moldeado por la hegemonía de los sectores dominantes, al decir de Gramsci (Kohan, 2007). 


\section{La articulación entre la deshistorización en el ejercicio del poder capitalista y la domina- ción comunicativa en los intercambios entre modelos de cultura o sociedad.}

A continuación aportaremos precisión al razonamiento esencial sobre la articulación propuesta, subrayando que ambos componentes se complementan en perturbaciones culturales, impuestas por el poder burgués, cuyo "driver" es un modo de dominación capaz de activar a esa deshistorización de la única manera posible: la comunicativa. Abstraída una de la otra, es improbable que "funcionen" con eficiencia para intentar contrarrestar a la dinámica impredecible de la cultura.

¿Cómo se describirían las consecuencias de la sumisión de los individuos subjetivados a dicha articulación? La respuesta, la recibimos desde Bourdieu (2000), quien advierte que en el seno de las sociedades más ricas el dualismo reposa en la distribución desigual del "capital cultural", generando un verdadero "racismo de la inteligencia" o del discernimiento pertinente. Los pobres ya no son oscuros ni haraganes, sino imbéciles e incultos. Los excluidos son confinados al refugio de la nacionalidad y el nacionalismo.

Esa articulación dilucida quiénes están dentro y quiénes fuera del centro de dominio capitalista. Este es un efecto esencial de los procesos comunicativos para la subordinación de lo, y de los inferidos como no-cultura, o ahistórico(s). La asimetría comunicativa es ineludible. La implicación política de las consecuencias de la articulación enunciada en tanto perturbación comunicativa debería saltar a la vista. No es posible confrontarla si no es colocándose comunicativamente del lado contrario a esa dominación.

Teniendo en cuenta que el sometimiento a la deshistorización en el ejercicio del poder capitalista en articulación con la dominación comunicativa pre-infiere a los otros fuera de la autoproducción burguesa como no-cultura, incultos por ahistóricos, ante tal contingencia, es necesaria una actitud crítica para, la construcción de otra forma de hacer política que involucre otra semiótica; construir otro sistema de significaciones, distinto y opuesto al sistema dominante. El desafío cultural para los oprimidos es más complejo que solamente resignificar o cambiar los códigos existentes. Ahí comienza la subversión, que se profundiza cuando rompe el "orden natural" o "civilizado"; cuando los "incultos" cuestionan la existencia misma de los opresores (Pineda, 2010).

Profundizando en el análisis crítico pertinente, entre los procesos que componen la raíz de la articulación entre la deshistorización en el ejercicio del poder burgués y 
la dominación comunicativa, primeramente expondremos al de la objetualización del sujeto. Acanda (1998) recuerda que La Ilustración fue la expresión histórica de la ideología burguesa de la emancipación, y abrió pasó a la interpretación de la realidad como algo racional, por lo tanto explicable. Su objetivo era el de liberar a los hombres del engaño y la superstición mediante la luz del saber, y convertirlos así, de esclavos en señores y dueños de su vida. Decía Kant, la Ilustración es la liberación del hombre de su culpable incapacidad. Que significa la imposibilidad de servirse de su propia inteligencia sin la guía de otro. Esta incapacidad es culpable porque su causa no reside en la falta de inteligencia sino de decisión y valor para servirse por sí mismo de ella sin la tutela de otro porque "la esencia de la Ilustración era la voluntad de que el individuo alcanzara la madurez, la capacidad de autonomía y responsabilidad en la dirección de su propia vida" (Acanda, 1998: 1).

Aspiración, posible de materializar mediante el intercambio subjetivo simétrico del saber mutuo, la memoria común y los programas para el control de la conducta humana contenidos en sistemas semióticos o comunicativos. El propósito teórico de la Ilustración era concebir al hombre como sujeto de su vida. Sujeto en tanto convierte en objetos de su actividad a los fenómenos que lo rodean, no se subordina ya a ellos (sean fuerzas naturales o instituciones sociales) sino que los objetualiza para dominarlos y utilizarlos en la conformación de una vida feliz. Y es aquí donde aparecen las contradicciones insalvables del proyecto de cambio social que se planteó la Ilustración. Y si bien, el objetivo era educar a los hombres (Acanda, 1998), ¿quién introduce la luz en la mente de los hombres? Otros hombres: los ya ilustrados. Ellos convierten a los demás individuos en objetos de su actividad educativa, y son los que los conducen hacia la razón y la felicidad. Es decir, en la actividad de ilustración, se objetualiza al otro.

Si tras analizar el proceso anterior recordamos que la clase burguesa, que emergió revolucionaria, mutó en conservadora a partir de 1848, al lograr el control del poder. Imponiendo como sujeto social dominador (colectivo) una nueva reproducción acorde a sus intereses, y designándose a sí misma como base ineludible de una meritocracia controladora de la expansión sistémica del capitalismo a escala mundial, podríamos abrirnos paso hacia la comprensión de estrategias de objetualización de los sujetos de clases subalternas, por parte de la burguesía; aprovechando una grave insuficien- 
cia teórico-conceptual de la propia Ilustración: las relaciones intersubjetivas (sujetosujeto) sólo pueden ser comprendidas en los marcos de la Ilustración clásica como relaciones objetuales (sujeto-objeto). Así "la Ilustración se traiciona a sí misma. Divide a los hombres en dos grupos: los educadores y los educados. La aspiración a la autodeterminación cabe tan sólo para los sujetos ilustradores, no para los individuosobjetos a ser ilustrados. Su función emancipadora cae prisionera de su tendencia objetualizante y cosificadora” (Acanda 1998: 2).

Entonces, la intersubjetivación -de los individuos que representan a un modelo- a través de sistemas semióticos manipulados por la hegemonía angloestadounidense "se configura bajo el signo de la dominación porque la burguesía desarrolla su proceso de cambio social conservando la dominación, la asimetría de las relaciones sociales intersubjetivas, y desarrollando hasta el paroxismo el proceso de objetualización o cosificación” (Acanda, 1998:2).

La cosificación, como recurso de la dominación comunicativo, es zócalo de la deshistorización en el ejercicio del poder burgués. Cuando el sujeto social dominado (colectivo) intelige su "inferioridad" en relación con el modelo hegemónico en tanto cultura, o cotidianidad capitalista, se sumerge en un aprendizaje ahistórico de los "textos" deformados por el código hegemónico. La intersubjetivación mediante sistemas comunicativos manipulados:

impone una coerción que se instituye por medio del reconocimiento [semantización] extorsionado que el dominado no puede dejar de prestar al dominante al no disponer, para pensarlo y pensarse, más que de instrumentos de conocimiento que tiene en común con él y que no son otra cosa que la forma incorporada de la relación de dominio (Bourdieu, 2003:8).

Los modelos de cultura o sociedad, sometidos a la asimetría que impone el modelo angloestadounidense, sufren un proceso de cosificación que se agudiza al semantizar (consumir) en sus interacciones "textos" propios y ajenos deformados por el código de dicha hegemonía.

Durante la deshistorización en el ejercicio del poder burgués se "comunica" semantizando, desde la subversión de las competencias sintáctica y semántica o mecanismos de regulación comunicativa que ambas integran. La asimetría del proceso de intersubjetivación 
(autoproducción) de los individuos dentro de un modelo de cultura o sociedad, mediante sistemas semióticos o comunicativos que soportan al modelo, ha sido y es indispensable al sujeto social dominador (colectivo) capitalista para imponer condiciones óptimas para el consenso a favor de sus intereses durante la pugna de fuerzas (poder). No olvidemos, que:

todas las clases sociales dominantes hasta ahora han sometido al resto de la sociedad a su modo específico de apropiación. Es decir, a su modo de autoproducción. Todas las clases dominantes existentes han logrado ese dominio porque se han auto-producido como los únicos sujetos verdaderos del proceso histórico, creando a todas las demás clases y grupos sociales como objetos de su auto-producción (Acanda, 1998: 2).

Objetualizar a los otros modelos e individuos subjetivados que los representan es una condición sine qua non de ese control asimétrico comunicativo en las interacciones entre modelos y con el hegemónico. Es improbable deshistorización alguna del poder sin cumplir esa condición, eminentemente subjetiva. De ahí que el sujeto social dominador (colectivo), la clase burguesa pese a su carácter históricamente revolucionario, no rebasó este patrón. Su modo de apropiación es enajenante y explotador porque implica que para auto-producirse como clase dominante tiene que mantener al resto de los grupos y clases sociales como objeto de su dominio; desprovisto de toda posibilidad de auto-determinación. El resto de la sociedad es solo un conjunto de objetos de su auto-reproducción (Acanda, 1998).

Lo que equivale a cosificar, en tanto hegemonía cultural de turno, y objetualizar en sus interacciones a modelos y sujetos otros, es una circunstancia inherente a la deshistorización del poder capitalista como recurso comunicativo conque trastornar al proceso de autoproducción.

Durante la deshistorización en el ejercicio del poder capitalista los individuos se subjetivan, a partir de apriorismos que se ocultan a través de recursos como la reacción instintiva ante necesidades prefabricadas desde esa deformación de "textos", con el código hegemónico que controla los flujos comunicativos durante los intercambios entre saber mutuo y memoria común, condicionados por sus intereses. Este proceso de intersubjetivación, se complica con las contradicciones epistemológicas provenientes de discursos teóricos "neutrales" sobre comunicación, cuando al individuo se le enseñan respuestas. Se le proporciona un saber meramente instrumental, un saber ope- 
rar con instrumentos cognoscitivos para encontrar las respuestas adecuadas en cada situación concreta a determinadas preguntas fijadas de antemano. Las preguntas se le presentan como demandas naturales e inescrutables, emanadas de la vida misma. Pero no se le enseña a interrogarse sobre esos instrumentos que se le proporcionan, y muchísimo menos a cuestionarse la racionalidad e intencionalidad de las preguntas, de las demandas que supuestamente debe resolver, los roles sociales que debe cumplir, las exigencias que debe satisfacer (Acanda, 1998).

Aun si complejo, debemos asumir que, "al afirmar que la propiedad privada nos ha vuelto tan estúpidos y unilaterales que consideramos que un objeto es nuestro sólo cuando lo poseemos físicamente, cuando lo consumimos, Marx destacó el carácter enajenante de una sociedad que lleva a patrón de toda la relación el vínculo entre el individuo aislado y la mercancía” (Acanda, 2002: 214-215).

A tenor de la mutación neoliberal del poder en el sistema burgués, mediante la recursividad comunicativa el sujeto histórico y la propia Historia -acumulación o totalidad culturaldevienen mercancía en función de la acumulación parasitaria de plusvalía, que sostiene la insoportable polarización en la distribución de la riqueza y del poder que la legítima, como manifestación del ejercicio deshistorizado del poder.

La deshistorización en el ejercicio del poder capitalista, en su definitiva implementación teórico-analítica, es consecuencia del redimensionamiento cultural capitalista que desde la modélica cultural antes enunciada impone lo relacional subjetivo, que se expande como componente indispensable de esta hegemonía. Puede encontrarse una diversidad de interpretaciones de la deshistorización del poder burgués, en estrecho vínculo con la evolución de la praxis en las relaciones subjetivas capitalistas.

\section{Conclusiones}

En el ejercicio de la deshistorización del poder radica la "eficiencia" que a través de la dominación comunicativa impone el modelo hegemónico, mediante la deformación de "textos" con su código, aparentando un orden político "eterno" y sin historia, del que los individuos subjetivados puedan auto-dilucidarse “deudores" en su noción del sentido.

El modelo angloestadounidense durante los flujos comunicativos entre sistemas semióticos es, mediante la acumulación burguesa de los signos (Buen Abad, 2009), 
un tejido ("textualidad") de re/semantizaciones fetichistas, en busca de imponer consenso global para la dominación en asimetría comunicativa, que es cultural; porque dependerá de esos sistemas semióticos para la adhesión del sujeto social dominado (colectivo) al modelo hegemónico, debido al control que el sujeto social dominador (colectivo) detenta sobre el ámbito de poder.

En un mundo subordinado a la supremacía cultural angloestadounidense, a través del trastorno y la banalización de la intersubjetivación y la producción social de signos, en una diversidad considerable de modelos de cultura o sociedad que interactúan a través de la dominación comunicativa, hasta el mismo Marx terminaría con todos sus huesos rotos, al decir de Kohan. No debemos perder de vista que ese sarcasmo puede resultar una herramienta útil para desfetichizar determinados entuertos construidos con delectación por la articulación en estudio.

Buen Abad (2010) nos alerta acerca de la necesidad de evaluar cuánta debilidad o cuanta ingenuidad hemos tenido a la hora de la lucha comunicacional y cuánto nos ha perjudicado nuestro error de la improvisación, el pragmatismo, el empirismo y la carencia de planificación comunicacional en el corto, el mediano y el largo plazo. Durante la emergencia de revoluciones que intentan materializar procesos emancipatorios, el sentido común entra en una crisis integral dentro de la pugna entre clases antagónicas, como sucede en Venezuela con la emergencia de la revolución bolivariana.

Precisamente la aguda y secular desigualdad que genera la dominación cultural capitalista, por comunicativa, obliga a su hegemonía actual a recurrir a lo deshistorizado como mecanismo funcional que mantenga su poder sobre los flujos comunicativos, sus soportes sistémicos y contenidos que transitan en su interior.

Esta investigación, solo abarca una porción -aun si importante- del problema del estudio del poder en los estudios sobre la comunicación, desde la perspectiva de la semiótica de la cultura. La articulación metódica propuesta nos permite confirmar que los modelos de cultura o sociedad, sometidos a la asimetría que impone el modelo angloestadounidense, desde una hegemonía (comunicativa) que se extiende en el tiempo y la dimensión espacial, más de lo soportable, sufren un proceso de cosificación que se agudiza al objetualizar en sus interacciones a modelos y sujetos a través de "textos" propios y ajenos deformados por el código de dicha hegemonía. 


\section{Bibliografía}

CACANDA, Jorge Luis (1998). "Educación, Ciencias Sociales y Cambio Social". En: Biblioteca Virtual de Filosofía y Pensamiento Cubano. Consultado el 12 de diciembre de 2009, desde http://biblioteca.filosofia.cu/

ACANDA, Jorge Luis (2001). "Capitalismo y racionalidad: una reflexión sobre la deuda externa desde el pensamiento crítico". En: Biblioteca Virtual de Filosofia y Pensamiento Cubano. Consultado el 5 de mayo 2008, desde http://biblioteca.filosofia.cu/

ACANDA, Jorge Luis (2002). Sociedad Civil y Hegemonía. La Habana: Centro Juan Marinello.

ALVARADO, Ramón (1991). Géneros y estrategias del discurso. Versión 1, 77-102.

AMIN, Samir (2005). "Imperialismo y Globalización". En: Centro de Estudios Miguel Enríquez-Archivo Chile. Consultado el 25 de mayo de 2007, desde www.archivochile.com/Imperialismo/doc_poli.../USdocimperial0004.pdf

AMIN, Samir (2010). "La desigualdad actual es el hecho social e histórico más impresionante de la historia". En: La Haine.org. Consultado el 12 de octubre de 2010, desde http://www.lahaine.org/blog.php?disp=impr\&blog=1\&p=48472

ARRIGHI, Giovanni (1997). "La globalización, la soberanía estatal y la interminable acumulación del capital”. En: Cholonautas. Consultado el 8 de febrero de 2006, desde http:// www.cholonautas.edu.pe/modulo/upload/ACUMULACION\%20DE\%20CAPITAL.pdf

BOURDIEU, Pierre (2000). "Los pobres ya no son haraganes, sino incultos". En: Página/12. Consultado el 3 de octubre de 2008, desde http://www.pagina12.com. ar/2000/00-07/00-07-03/univer01.htm

BOURDIEU, Pierre (2003). "La dominación masculina". En: Cholonautas. Consultado el 22 de enero de 2007, desde http://www.cholonautas.edu.pe/modulo/upload/ LADOMINACIONMASCULINA-BOURDIEU.pdf

BUEN ABAD DOMÍNGUEZ, Fernando (2009). "La ofensiva semiótica burguesa incluso con bases militares. Muchos medios, un sentido". En: Rebelion.org. Consultado el 30 de noviembre de 2010, desde http://www.rebelion.org/noticia.php?id=90334

BUEN ABAD DOMÍNGUEZ, Fernando (2010). "Reflexiones comunicacionales sobre las elecciones venezolanas". En: Rebelion.org. Consultado el 30 de septiembre de 2010, desde http://www.rebelion.org 
BUEN ABAD DOMÍNGUEZ, Fernando (2013a). "El problema de la Democracia es también un problema de Comunicación”. En Rebelion.org. Consultado el 28 de julio de 2013, desde http://www.rebelion.org/noticia.php?id=171668

BUEN ABAD DOMÍNGUEZ, Fernando (2013b). "El Capitalismo, su "Educación" y sus "Educadores". En: Rebelión.org. Consultado el 20 de septiembre de 2013, desde http://www.rebelion.org/noticia.php?id=135221

CABANILLES, Antonia (2004). "Semiótica de la cultura: los modelos de autodescripción. Entretextos”. En: Entretextos. Revista Electrónica Semestral de Estudios Semióticos de la Cultura No. 3, pp. 1-6. http://wwwugr.es/local/mcaceres/entretextos.htm.

CASTRO, Olalla (2004). "Cuando el centro del sistema absorbe a la periferia: la evolución del rap a través de la semiótica de la cultura”. En: Entretextos. Revista Electrónica Semestral de Estudios Semióticos de la Cultura No. 4, pp. 1-9. http://www.ugr.es/local/ mcaceres/entretextos.htm

CECEÑA, Ana Esther (2011). “¿Hegemonía o emancipación?”. En: Rebelion.org Consultado el 5 de enero de 2011, desde http://www.rebelion.org/noticia.php?id=140986

COLUSSI, Marcelo, Oscar R. Amado, y Rodolfo Solana (2011). "Los medios alternativos y las redes sociales". En: Rebelion.org. Consultado el 22 de diciembre de 2011, desde http://www.rebelion.org/noticia.php?id=141731

ERREJÓN, Iñigo (2010). "Construcción de poder político y hegemonía nacional-popular indígena en Bolivia”. En: Sumarios ISOC - Sociología y CC. Políticas. Consultado el 25 de abril de 2012, desde http://www.ceps.es/media/txt/papelestrabajo5.pdf

GALEANO, Eduardo (1999). Patas Arriba. Siglo XXI, España: Editores S.A.

GANDARILLA, José Guadalupe (2005). "Subversión (no sólo) de los saberes hegemónicos y la construcción de alternativas”. En: ALAI, América Latina en Movimiento. Consultado el 22 de abril de 2008, desde http://alainet.org/active/7955\&lang=es

GARCÍA LINERA, Álvaro (2011). La potencia plebeya. La Habana, Cuba: Fondo Editorial Casa de las Américas.

GÓMEZ, Víctor Andrés (2011a). "Signo del prejuicio en un modelo de cultura: imagen versus semejanza”. En: Temas, No. 65, pp. 85-93.

GÓMEZ, Víctor Andrés (2011b). "La dominación comunicativa, un desafío para el socialismo del siglo XXI”. En: Pensar a Contracorriente, No.VIII, pp. 194-220. 
GONZÁLEZ, Antonio y Paula González. "Imperialismo lingüístico". En: Servicios Kolnonia. Agenda Latinomaericana. Consultado el 22 de abril de 2007, desde http:// www.servicioskoinonia.org/agenda/archivo/obra.php?ncodigo=137

GRIMALDI HERRERA, Carmen (2009). "Anglofonía en el mundo actual: el inglés británico y el inglés americano". En: Contribuciones a las Ciencias Sociales. Consultado el 4 de enero de 2010, desde http://www.eumed.net/rev/cccss/06/cgh14.htm

GUEVARA, Alfredo (2011). " ¡La Transición en Cuba es del Disparate al Socialismo!" En: Kaos en la Red. Consultado el 26 de junio de 2011, desde http://www.kaosenlared.net

HAIDAR, Julieta (2003). "Las propuestas de Lotman para el análisis cultural y su relación con otras tendencias actuales”. Entretextos. Revista Electrónica Semestral de Estudios Semióticos de la Cultura, No. 4, http://www.ugr.es/local/mcaceres/entretextos.htm

KOHAN, Néstor (2003). Marx en su Tercer mundo. Hacia un socialismo no colonizado. La Habana, Cuba: Centro Juan Marinello.

KOHAN, Néstor (2007). "Gramsci y Marx. Hegemonía y poder en la teoría marxista”. En: La Haine.org. Libros Libres. Consultado el 22 de mayo de 2007, desde http:// www.amauta.lahaine.org.

KOHAN, Néstor (2011). "Ni calco ni copia. Ensayos sobre el marxismo argentino y latinoamericano". En: La Haine.org. Libros Libres. Consultado el 3 de enero de 2012, desde http://www.amauta.lahaine.org

LAMPIS, Mirko (2008). "Semiótica de la Cultura y Ciencias de la Inteligencia: un acercamiento interdisciplinario". Tesis de Doctorado. Universidad de Granada.

LEET, Patrick (2003). Comunidad en los Estados Unidos. En: Caminos, No. 27, pp. 16-25

LOTMAN, Iuri M. (2003). "La semiótica de la cultura y el concepto de texto". En: Entretextos Revista Electrónica Semestral de Estudios Semióticos de la Cultura, No. 2, http://www.ugr.es/\%7Emcaceres/Entretextos/entretextos2.htm

LOZANO, Jorge (1998). "La semioesfera y la teoría de la cultura". En: Espéculo, No. 8, pp. 1-7. http://pendientedemigracion.ucm.es/info/especulo/numero8/lozano.htm

MARTÍN-CABRERA, Luis (2010). "Reflexiones sobre el racismo anti-inmigrante en Estados Unidos”. En: Rebelion.org. Consultado el 22 de enero de 2010, desde http:// www.rebelion.org/mostrar.php?tipo $=5 \&$ id $=$ LuisMartínCabrera\&inicio $=0$ 
MARTÍNEZ HEREDIA, Fernando (2001). El corrimiento hacia el rojo. La Habana, Cuba: Editorial Ciencias Sociales.

MARTÍNEZ HEREDIA, Fernando (2003). "Imperialismo, guerra y resistencia". En: Temas, No. 33-34, pp. 103-111.

MARTÍNEZ HEREDIA, Fernando (2005). En el horno de los 90. La Habana, Cuba: Editorial Ciencias Sociales.

MELGAR BAO, Ricardo (2003). "Entre lo sucio y lo bajo: identidades subalternas y resistencia cultural en América Latina”. En: Temas No. 35, pp. 28-42.

MUÑOZ, Manuel (2009). "El Supremacismo Lingüístico". En Rebelio.org. Libros Libres. Consultado el 28 de junio de 2009, desde http://www.rebelion.org.

NAVARRO, Desiderio (2007). "La cultura de masas, semiótica, sociología y praxis social". En: A pe(n)sar de todo. Para leer en contexto. La Habana, Cuba: Editorial Letras Cubanas.

PETRAS James (2012). "Política del lenguaje”.En: Rebelión.org. Consultado El 25 de mayo de 2012, desde http://www.rebelion.org/noticia.php?id=150182.

PINEDA, Francisco (2009). "Otra semiótica para otra política”. En: Entretextos. Revista Electrónica Semestral de Estudios Semióticos de la Cultura, 14-15-16, 78-83.

http://www.ugr.es/ mcaceres/entretextos/entre14-16/pineda.html

ROMANO, Vicente (2004). "La formación de la mentalidad sumisa". En: Rebelión. org. Libros Libres. Consultado el 22 de enero de 2006, desde http://www.rebelión.org.

ROMANO, Vicente (2005). "La intoxicación lingüística. El uso perverso de la lengua”. En: Rebelión.org. Libros Libres. Consultado el 20 de febrero de 2011, desde http://www.rebelion.org

SÁNCHEZ NORIEGA, José Luis (2007). "Dominación Comunicativa del Tercer Mundo y sensibilización para la solidaridad”. En: Foro Ignacio Ellacuría. Sociedad y Cristianismo: La globalización y sus excluidos, 2da edición.

SERRANO, María José (2011). Sociolingüística. Barcelona: Ediciones del Serbal.

SONESSON, Göran (2002). "Dos modelos de la globalización”. En: Criterios No. 33, pp. 107-131.

SPIVAK, Gayatri Chakravorty (1996). "Estudios de la Subalternidad: Deconstruyendo la Historiografía". En: Cholonautas. Consultado el 5 de mayo de 2005, 
desde http://www.cholonautas.edu.pe

SPIVAK, Gayatri Chakravorty (1998). “¿Puede hablar el subalterno?”. En: Orbis Tertius, III (6), pp. 1-44.

TENOCH, Alfredo (2006). De la traducción intersemiótica a la competencia intersemiótica. Versión 18, pp. 115-132.

VÁSQUEZ ROCCA, Adolfo (2005). "La moda en la postmodernidad. Deconstrucción del fenómeno fashion”. En: Nómadas, No. 11, pp. 169-176.

ZINN, Howard (2004). La otra Historia de los Estados Unidos. La Habana, Cuba: Editorial Ciencias Sociales. 